\title{
Global Analysis of SIRS Epidemic Model With General Incidence Function and Incomplete Recovery Rates Stochastical Model
}

\author{
Dramane Ouedraogo ${ }^{1}$, Ali Traore ${ }^{2}$, Aboudramane Guiro ${ }^{1}$ \\ ${ }^{1}$ Unité de Formation et de Recherche en sciences et Techniques, LAboratoire de Mathématiques et Informatique (LAMI), \\ Université Nazi Boni de Bobo-Dioulasso, 01 bp 1091, Bobo-Dioulasso 01, Burkina Faso \\ ${ }^{2}$ Unité de Formation et de Recherche en sciences et Techniques, LAboratoire de Mathématiques et Informatique (LAMI), \\ Université Joseph Ki Zerbo, Ouagadougou, Burkina Faso
}

Correspondence: Dramane Ouedraogo, Université Nazi Boni de Bobo-Dioulasso, 01 bp 1091, Bobo-Dioulasso 01, Burkina Faso. E-mail: dramaneouedraogo268@yahoo.ca

Received: September 26, 2020 Accepted: October 30, 2020 Online Published: November 19, 2020

doi:10.5539/jmr.v12n6p100 URL: https://doi.org/10.5539/jmr.v12n6p100

\begin{abstract}
In this paper, deterministic and stochastic models are developped for a class of SIRS epidemic models. Firstly, The conditions for the existence, local and global stability of the disease-free equilibrium and endemic equilibrium are obtained. Secondly, we built the stochastic model. The populations are computationally simulated under various conditions. Comparisons are made between the deterministic and stochastic model.
\end{abstract}

Keywords: SIRS epidemic model, equilibrium, local stability, general incidence function, stochastic differential equation

\section{Introduction}

Historically the mathematical modeling of epidemics has started since the time of Graunt (Graunt, 1662). In fact, Kermack and Mckendric (Kermack, Mckendric, 1927) describe some classical deterministic mathematical models of epidemiology by considering the total population into three classes namely of epidemiology susceptible (S) individuals, infected (I) individuals and recovered $(\mathrm{R})$ individuals which is known to us as SIR epidemic models. This SIR epidemic model is very important in today's analysis of diseases. When the recovered lost immunity we say that we are an SIRS epidemic models.

Epidemic models have been studied by many authors. Most of them are interesting in the formulation of the incidence rate, i.e. the infection rate of susceptible individuals through their contacts with infective (see, for example, (Gao,Chen, Nieto,Torres, 2006),(Kyrychko, Blyuss,2005),(Li, Wang, Wang,Jin, 2007)). In order to model the disease transmission process several authors employ the following incidence functions. The first one is the bilinear incidence rate $\beta S I$, where $S$ and $I$ are respectively the number of susceptible and infective individuals in the population, and $\beta$ is a positive constant ((Jiang, Wei, 2008, (Zhang, Li, Zhang 2008), (Zhou, Liu, 2003)). The second one is the saturated incidence rate of the form $\frac{\beta S I}{1+\alpha_{1} S}$, where $\alpha_{1}$ is a positive constant. The effect of saturation factor (refer to $\alpha_{1}$ ) stems from epidemic control (tacking appropriate preventive measures)((Wei,Chen, 2008), (Zhang, Jin, Liu, Zhang, 2008)).

Stochastic differential equation (SDE) model is a natural generalization of ordinary differential equation (ODE) model. SDE became increasingly more popular in mathematical biology ((Allen, 2003),(Gard 1988) and the references therein). In (Allen, Victory,2003), a SDE model for transmission of schistosomiasis was analyzed. That model assumes that births and deaths are neglected. So, the computational work is involved in a computation of $\sqrt{B} \eta$ that one requires other schemes in which we solve an initial value problem.

In this paper, we consider SIR model of disease transmission that was presented and studied in (Connell, McCuskey, 2010). It is a refinement and generalization of earlier model that used incidence function $\frac{\beta S I_{\tau}}{1+\alpha I_{\tau}}$. The model given in (Connell, McCuskey, 2010) allows for saturation in the force of infection by using the general incidence function $f(S, I)$. 
In this paper, we consider the following SIRS epidemic model described by differential equations.

$$
\left\{\begin{array}{l}
\dot{S}=B-\mu_{1} S-f(S, I)+\delta R, \\
\dot{I}=f(S, I)-\left(\mu_{2}+\gamma\right) I, \\
\dot{R}=\gamma I-\left(\mu_{3}+\delta\right) R,
\end{array}\right.
$$

with initial conditions:

$$
S(0)>0, \quad I(0)>0 \quad \text { and } \quad R(0)>0
$$

where $S(t), I(t)$ and $R(t)$ denote the numbers of susceptible, infective and recovered individuals at time t, respectively, $B$ is the recruitment rate of the population, $\mu_{i}(i=1,2,3)$ is the death rate of $S(t), I(t)$ and $R(t)$, respectively, $\gamma$ is the recovery rate of the infective individuals, $f(S, I)$ is the general incidence function, $\delta$ is the rate which the recovered individuals become susceptible again.

The organization of the paper is as follows. In Section 2, We give the positiveness and the boundedness of the different classes, the existence of equilibria is presented, We study the local and global stability of the free-equilibrium point and the global stability of the endemic equilibrium. We construct a Stochastic differential model in Section 3 and we derive an equivalent stochastic model. Section 4 is devoted to describe a numerical method to solve the equivalent stochastic model and numerical simulation. Finally, in Section 5, we end by a conclusion.

\section{Analysis of SIRS Model}

\subsection{Positiveness, Eventual Boundedness}

We consider the positiveness of system (1). We have the following basic lemma.

Lemma 1 For any solutions $(S(t), I(t), R(t))$ of system (1) with the initial conditions (2),

$$
S(t)>0, \quad I(t)>0 \text { and } R(t)>0, \text { for any } t \geq 0
$$

and there solutions are bounded.

Proof. First, by (2), we have that $S(0)>0, \quad I(0)>0$ and $\quad R(0)>0$ and by continuity of the solution of (1), we may assume that there exists a positive $t_{1}$ such that $S(t)>0, \quad I(t)>0$ and $R(t)>0$ for any $0 \leq t<t_{1}$. Suppose that there exists a positive $t_{1}$ such that $S\left(t_{1}\right)=0$ and $S(t)>0, \quad I(t)>0$ and $R(t)>0$ for any $0 \leq t<t_{1}$. But by (1), we have that $\frac{d S}{d t}\left(t_{1}\right) \geq B>0$ which is a contradiction to the fact $S(t)>0=S\left(t_{1}\right)$ for any $0 \leq t<t_{1}$. Thus, we have that if there exists a positive $t_{1}$ such that $S(t)>0, \quad I(t)>0$ and $R(t)>0$ for any $0 \leq t<t_{1}$, then $S\left(t_{1}\right)>0$.

Moreover, by (1), we have that

$$
\left\{\begin{array}{l}
I(t)=e^{-\left(\mu_{2}+\gamma\right) t} I(0)+e^{-\left(\mu_{2}+\gamma\right) t} \int_{0}^{t} e^{\left(\mu_{2}+\gamma\right) u}(f(S(u), I(u))) d u, \\
R(t)=e^{-\left(\mu_{3}+\delta\right) t} R(0)+e^{-\left(\mu_{3}+\delta\right) t} \int_{0}^{t} \gamma I(u) e^{\left(\mu_{3}+\delta\right) u} d u,
\end{array}\right.
$$

which implies that if there exists a positive $t_{1}$ such that $S(t)>0, \quad I(t)>0$ and $\quad R(t)>0$ for any $0 \leq t<t_{1}$, then we also obtain that $I\left(t_{1}\right)>0$ and $R\left(t_{1}\right)>0$. Thus, as a result, we obtain (3).

By doing the sum of the three equations in system (1), we have

$$
\frac{d(S+I+R)}{d t} \leq B-\bar{\mu}(S+I+R)
$$

where $\bar{\mu}=\min \left(\mu_{1}, \mu_{2}, \mu_{3}\right)$. By using (Guiro, Ouedraogo and Ouedraogo, 2018), we conlude that $S(t), I(t)$ and $R(t)$ are bounded.

\subsection{Existence of Equilibria}

For model (1) we introduce the following assumptions.

H1 All parameters $B, \mu_{i}(i=1,2,3), \delta$ and $\gamma$ are positive constants.

H2 Function $f(S, I)$ is continuously differentiable for all $S \geq 0, I \geq 0, f(S, 0)=f(0, I)=0$ and $f(S, I)>0$ for all $S>0$, $I>0$. 
Let us denote by $f_{1}$ and $f_{2}$ the partial derivatives of $f$ with respect to the first and to the second variable.

Let $R_{0}=\frac{f_{2}\left(S^{0}, 0\right)}{\left(\mu_{2}+\gamma\right)}$ where $S^{0}=\frac{B}{\mu_{1}}$.

Remark $1 R_{0}$ is the basic reproduction number evaluate the average number of new infections generated by a single infected individual in a completely susceptible population.

On the existence of the nonnegative equilibria of model (1), we have the following results:

\section{Theorem 1}

(1) If $R_{0} \leq 1$, then model (1) has an unique disease-free equilibrium $E_{0}$.

(2) If $R_{0}>1$, then model (1) has an unique endemic equilibrium.

Proof. Let $E=(S, I, R)$ be an equilibrium point of system (1).

By using the third equation of (1), we get

$$
R=\frac{\gamma I}{\mu_{3}+\delta}
$$

By adding the first and the second equations of the model (1), we have

$$
S=\frac{B}{\mu_{1}}-\left(-\frac{\delta \gamma}{\left(\mu_{3}+\gamma\right) \mu_{1}}+\left(\mu_{2}+\gamma\right)\right) I
$$

so

$$
S=S^{0}-\theta I
$$

where $\theta=\frac{-\delta \gamma}{\left(\mu_{3}+\gamma\right) \mu_{1}}+\left(\mu_{2}+\gamma\right)$. By using the second equation of (1), we get

$$
\frac{f\left(S^{0}-\theta I, I\right)}{I}=\left(\mu_{2}+\gamma\right)
$$

Let $\Phi(I)=\frac{f\left(S^{0}-\theta I, I\right)}{I}-\left(\mu_{2}+\gamma\right)$

$$
\lim _{I \rightarrow 0^{+}} \Phi(I)=-\theta f_{1}\left(S^{0}, 0\right)+f_{2}\left(S^{0}, 0\right)-\left(\mu_{2}+\gamma\right)
$$

since $f(S, 0)=0$, we have

$$
\lim _{I \rightarrow 0^{+}} \Phi(I)=\left(\mu_{2}+\gamma\right)\left(R_{0}-1\right)
$$

and we have also $\Phi(\bar{I})=-\left(\mu_{2}+\gamma\right)$ with $\bar{I}=\frac{S^{0}}{\theta}$. When $R_{0} \leq 1$, we have $\lim _{I \rightarrow 0^{+}} \Phi(I) \leq 0$, thus, there is not any $I^{*}>0$ such that $\Phi\left(I^{*}\right)=0$. Therefore system (2) have a unique free-disease equilibrium $E_{0}$.

When $R_{0}>1$, we have $\lim _{I \rightarrow 0^{+}} \Phi(I) \geq 0$ so there exists $\left.I^{*} \in\right] 0, \bar{I}[$. This implies that system (2) have a unique endemic equilibrium point $E^{*}$.

\subsection{Stability of the Disease-Free Equilibrium for $R_{0} \leq 1$}

In this section, we study the local and global behaviour of the disease-free equilibrium.

Theorem 2 The disease-free equilibrium is locally asymptotically stable if $R_{0} \leq 1$

Proof. The characteristic equation of linearized system (1) at $E_{0}$ gives the following equation,

$$
\left(-\mu_{3}-\delta-\lambda\right)\left[\left(-\mu_{1}-f_{1}\left(S^{0}, 0\right)-\lambda\right)\left(f_{2}\left(S^{0}, 0\right)-\left(\mu_{2}+\gamma\right)-\lambda\right)+f_{1}\left(S^{0}, 0\right) f_{2}\left(S^{0}, 0\right)\right]=0 .
$$

It is exact to check that all solutions $\lambda$ of equation (4) are a negative real parts.

Indeed, the equation (4) has negative root $\lambda=-\mu_{3}-\delta$ and other roots are given by

$$
\left(-\mu_{1}-f_{1}\left(S^{0}, 0\right)-\lambda\right)\left(f_{2}\left(S^{0}, 0\right)-\left(\mu_{2}+\gamma\right)-\lambda\right)+f_{1}\left(S^{0}, 0\right) f_{2}\left(S^{0}, 0\right)=0 .
$$


By developping (5), we get

$$
\lambda^{2}+\left(\mu_{1}+f_{1}\left(S^{0}, 0\right)-f_{2}\left(S^{0}, 0\right)+\mu_{2}+\gamma\right) \lambda-\mu_{1} f_{2}\left(S^{0}, 0\right)+\mu_{1}\left(\mu_{2}+\gamma\right)+f_{1}\left(S^{0}, 0\right)\left(\mu_{2}+\gamma\right)=0 .
$$

Since $f(S, 0)=0$, we have

$$
\lambda^{2}+\left(\mu_{1}-f_{2}\left(S^{0}, 0\right)+\mu_{2}+\gamma\right) \lambda-\mu_{1} f_{2}\left(S^{0}, 0\right)+\mu_{1}\left(\mu_{2}+\gamma\right)=0 .
$$

Since $R_{0} \leq 1$, we obtain

$$
\mu_{1}-f_{2}\left(S^{0}, 0\right)+\mu_{2}+\gamma>0 .
$$

Therefore, by the Routh-Hurwitz criterion all the roots of equation (7) have a negative real parts. This shows that equilibrium $E_{0}$ is locally asymptotically stable. This completes the proof.

H3 For all $(S, I) \in \mathbb{R}^{2}, f(S, I) \leq f_{2}\left(S^{0}, 0\right) I$.

Theorem 3 The disease-free equilibrium is globally asymptotically stable if $R_{0} \leq 1$

Proof. The proof is based on using a comparison theorem (Lakshmikantham, Leela, Martynyuk, 1989). Note that the equations of infected components in system (1) can be expressed as

$$
\dot{I} \leq\left(f_{2}\left(S^{0}, 0\right)-\left(\mu_{2}+\gamma\right)\right) I .
$$

So, we deduce that, $f_{2}\left(S^{0}, 0\right)-\left(\mu_{2}+\gamma\right)$ is negative since $R_{0} \leq 1$.

Thus, $I(t) \rightarrow 0$ as $t \rightarrow \infty$ for the system (8). Consequently, by a standard comparison theorem (Lakshmikantham, Leela, Martynyuk, 1989), $I(t) \rightarrow 0$ as $t \rightarrow \infty$ and substituting $I=0$ into system (1) $S \rightarrow S^{0}$ as $t \rightarrow \infty$.

Thus, $(S, I, R) \rightarrow\left(S^{0}, 0,0\right)$ as $t \rightarrow \infty$ for $R_{0} \leq 1$. Therefore, $E_{0}$ is globally asymptotically stable if $R_{0} \leq 1$.

2.4 Stability of the Endemic Equilibrium for $R_{0}>1$

In this section, we study the global dynamics for $R_{0}>1$.We make this additional assumption as in (Guiro, Ngom, Ouedraogo, 2017).

H4 For all $(S, I) \in \mathbb{R}_{+}^{2}$,

$$
\frac{f\left(S^{*}, I^{*}\right)}{f(S, I)} \leq \frac{S^{*}}{S} \leq \frac{I^{*}}{I}
$$

We recall that the endemic equilibrium $E^{*}$ exists if and only if $R_{0}>1$.

Theorem 4 If $R_{0}>1$ the endemic equilibrium $E^{*}$ is globally asymptotically stable.

Proof. We consider the system (1) when $R_{0}>1$, there exists a unique endemic equilibrium $E^{*}$. We now establish the global asymptotic stability of this endemic equilibrium.

Evaluating both sides of (1) at $E^{*}$ gives

$$
\left\{\begin{array}{l}
B=\mu_{1} S^{*}+f\left(S^{*}, I^{*}\right)-\delta R^{*}, \\
f\left(S^{*}, I^{*}\right)=\left(\mu_{2}+\gamma\right) I^{*}, \\
\gamma I^{*}=\left(\mu_{3}+\delta\right) R^{*}
\end{array}\right.
$$

Let

$$
g(x)=x-1-\ln x
$$

and

$$
\begin{aligned}
V_{S} & =g\left(\frac{S}{S^{*}}\right) \\
V_{I} & =g\left(\frac{I}{I^{*}}\right), \\
V_{R} & =g\left(\frac{R}{R^{*}}\right) .
\end{aligned}
$$


Thus, $V_{S} \geq 0, \quad V_{I} \geq 0, \quad V_{R} \geq 0$ with equality if and only if $S=S^{*}, I=I^{*}$ and $R=R^{*}$. We will study the behaviour of the Lyapunov function

$$
V(t)=V_{S}+V_{I}+V_{R}
$$

We can see that $V(t) \geq 0$ with equality if and only if $S=S^{*}, I=I^{*}$ and $R=R^{*}$.

The derivatives of $V_{S}, V_{I}$, and $V_{R}$ will be calculated separately and then combined to get the desired quantity $\frac{d V}{d t}$.

$$
\begin{aligned}
\frac{d V_{s}}{d t} & =\frac{1}{S^{*}}\left(1-\frac{S^{*}}{S}\right) \frac{d S}{d t} \\
& =\frac{1}{S^{*}}\left(1-\frac{S^{*}}{S}\right)\left(B-\mu_{1} S-f(S, I)+\delta R\right) .
\end{aligned}
$$

Using the first equation of (10) to replace $B$ gives

$$
\begin{aligned}
\frac{d V_{s}}{d t} & =\frac{1}{S^{*}}\left(1-\frac{S^{*}}{S}\right)\left(\mu_{1}\left(S^{*}-S\right)+\left(f\left(S^{*}, I^{*}\right)-f(S, I)\right)+\delta\left(R-R^{*}\right)\right) \\
& =-\frac{\mu_{1}}{S S^{*}}\left(S-S^{*}\right)^{2}+\frac{f\left(S^{*}, I^{*}\right)}{S^{*}}\left(1-\frac{S^{*}}{S}\right)\left(1-\frac{f(S, I)}{f\left(S^{*}, I^{*}\right)}\right)+\frac{\delta R^{*}}{S^{*}}\left(1-\frac{S^{*}}{S}\right)\left(\frac{R}{R^{*}}-1\right) .
\end{aligned}
$$

Then, we may write

$$
\frac{d V_{s}}{d t}=-\frac{\mu_{1}}{S S^{*}}\left(S-S^{*}\right)^{2}+\frac{f\left(S^{*}, I^{*}\right)}{S^{*}}\left(1-\frac{f(S, I)}{f\left(S^{*}, I^{*}\right)}-\frac{S^{*}}{S}+\frac{S^{*} f(S, I)}{S f\left(S^{*}, I^{*}\right)}\right)+\frac{\delta R^{*}}{S^{*}}\left(1-\frac{S^{*}}{S}\right)\left(\frac{R}{R^{*}}-1\right)
$$

Next, we calculate $\frac{d V_{I}}{d t}$.

$$
\begin{aligned}
\frac{d V_{I}}{d t} & =\frac{1}{I^{*}}\left(1-\frac{I^{*}}{I}\right) \frac{d I}{d t} \\
& =\frac{1}{I^{*}}\left(1-\frac{I^{*}}{I}\right)\left(f(S, I)-\left(\mu_{2}+\gamma\right) I\right) \\
& =\frac{1}{I^{*}}\left(1-\frac{I^{*}}{I}\right)\left(f\left(S^{*}, I^{*}\right) \frac{f(S, I)}{f\left(S^{*}, I^{*}\right)}-\left(\mu_{2}+\gamma\right) I^{*} \frac{I}{I^{*}}\right) .
\end{aligned}
$$

Using the second equation of (10) to replace $\left(\mu_{2}+\gamma\right) I^{*}$ gives

$$
\frac{d V_{I}}{d t}=\frac{f\left(S^{*}, I^{*}\right)}{I^{*}}\left(1-\frac{I^{*}}{I}\right)\left(\frac{f(S, I)}{f\left(S^{*}, I^{*}\right)}-\frac{I}{I^{*}}\right)
$$

We have,

$$
\frac{d V_{I}}{d t}=\frac{f\left(S^{*}, I^{*}\right)}{I^{*}}\left(\frac{f(S, I)}{f\left(S^{*}, I^{*}\right)}-\frac{I}{I^{*}}-\frac{I^{*} f(S, I)}{I f\left(S^{*}, I^{*}\right)}+1\right)
$$

After that, we evaluate $\frac{d V_{R}}{d t}$.

$$
\begin{aligned}
\frac{d V_{R}}{d t} & =\frac{1}{R^{*}}\left(1-\frac{R^{*}}{R}\right) \frac{d R}{d t} \\
& =\frac{1}{R^{*}}\left(1-\frac{R^{*}}{R}\right)\left(\gamma I-\left(\mu_{3}+\delta\right) R\right) \\
& =\frac{1}{R^{*}}\left(1-\frac{R^{*}}{R}\right)\left(\gamma I^{*} \frac{I}{I^{*}}-\left(\mu_{3}+\delta\right) R^{*} \frac{R}{R^{*}}\right) .
\end{aligned}
$$

Using the last equation of (10) to replace $\gamma I^{*}$ gives

$$
\frac{d V_{R}}{d t}=\frac{\gamma I^{*}}{R^{*}}\left(1-\frac{R^{*}}{R}\right)\left(\frac{I}{I^{*}}-\frac{R}{R^{*}}\right)
$$

We get,

$$
\frac{d V_{R}}{d t}=\frac{\gamma I^{*}}{R^{*}}\left(\frac{I}{I^{*}}-\frac{R}{R^{*}}-\frac{R^{*} I}{R I^{*}}+1\right)
$$


Combining equations (13)-(15),

$$
\begin{aligned}
\frac{d V}{d t} \leq & -\frac{\mu_{1}}{S S^{*}}\left(S-S^{*}\right)^{2}+\max \left\{\frac{f\left(S^{*}, I^{*}\right)}{S^{*}} ; \frac{\delta R^{*}}{S^{*}} ; \frac{\gamma I^{*}}{R^{*}}\right\} \\
& \times\left(2+\frac{S^{*} f(S, I)}{S f\left(S^{*}, I^{*}\right)}-\frac{S^{*} R}{S R^{*}}-\frac{I^{*} f(S, I)}{I f\left(S^{*}, I^{*}\right)}-\frac{R^{*} I}{R I^{*}}\right)
\end{aligned}
$$

by adding and substracting the quantity

$$
1+\ln \left(\frac{S^{*} f(S, I)}{S f\left(S^{*}, I^{*}\right)}\right)+\ln \left(\frac{I^{*} f(S, I)}{I f\left(S^{*}, I^{*}\right)}\right)+\ln \left(\frac{S^{*} R}{S R^{*}}\right)+\ln \left(\frac{R^{*} I}{R I^{*}}\right)
$$

we obtain

$$
\begin{aligned}
\frac{d V}{d t} \leq & -\frac{\mu_{1}}{S S^{*}}\left(S-S^{*}\right)^{2}+\max \left\{\frac{f\left(S^{*}, I^{*}\right)}{S^{*}} ; \frac{\delta R^{*}}{S^{*}} ; \frac{\gamma I^{*}}{R^{*}}\right\}\left(g\left(\frac{S^{*} f(S, I)}{S f\left(S^{*}, I^{*}\right)}\right)\right. \\
& -g\left(\frac{I^{*} f(S, I)}{I f\left(S^{*}, I^{*}\right)}-g\left(\frac{S^{*} R}{S R^{*}}\right)-g\left(\frac{R^{*} I}{R I^{*}}\right)\right),
\end{aligned}
$$

Since the function $g$ is monotone on each side of point 1 and is minimized at this point $1, \mathbf{H} 4$ implies

$$
g\left(\frac{S^{*} f(S, I)}{S f\left(S^{*}, I^{*}\right)}\right) \leq g\left(\frac{I^{*} f(S, I)}{I f\left(S^{*}, I^{*}\right)}\right) .
$$

Since $g \geq 0$, then

$$
\frac{d V}{d t} \leq 0
$$

for all $(S, I) \in \mathbb{R}_{+}^{2}$ with equality only for $S=S^{*}, I=I^{*}$ and $R=R^{*}$.

Hence, the endemic equilibrium $E^{*}$ is the only positively invariant set of the system $(1)$ contained in $\left\{(S, I) \in \mathbb{R}_{+}^{2} ; S=\right.$ $\left.S^{*}, I=I^{*} R=R^{*}\right\}$. Then, it follows that $E^{*}$ is globally asymptotically stable (Lasalle, 1976).

\section{Stochastic Model}

\subsection{Stochastic Differential Equation Model}

To derive a stochastic model, we apply a similar procedure to that described in (Allen, 1999). Here, we neglect the possibility of multiple events of order $(\Delta t)^{2}$. The possible changes in the populations over a short time $\Delta t$, concern individual births, deaths and transformation. These changes are produced in Table 1, together with their corresponding probability. Let's denote this change by $\eta=(\Delta S, \Delta I, \Delta R)^{T}$.

Neglecting terms of the order $(\Delta t)^{2}$, the mean of system (1) is given by

$$
E(\eta)=\sum_{i=1}^{8} P_{i} \eta_{i}=\left(\begin{array}{c}
B-\mu_{1} S-f(S, I)+\delta R \\
f(S, I)-\left(\mu_{2}+\gamma\right) I \\
\gamma I-\left(\mu_{3}+\delta\right) R
\end{array}\right) \Delta t=\mu \Delta t
$$

Further, the covariance matrix of system (1) is given by

$$
E\left(\eta \eta^{T}\right)=\sum_{i=1}^{8} P_{i} \eta_{i} \eta_{i}^{T}=\left(\begin{array}{lll}
B_{11} & B_{12} & B_{13} \\
B_{12} & B_{22} & B_{23} \\
B_{13} & B_{23} & B_{33}
\end{array}\right) \Delta t=B \Delta t,
$$

where

$$
\begin{aligned}
& B_{11}=B+\mu_{1} S+f(S, I)+\delta R, \\
& B_{12}=-f(S, I), \\
& B_{13}=-\delta R, \\
& B_{22}=f(S, I)+\left(\mu_{2}+\gamma\right) I, \\
& B_{23}=-\gamma I, \\
& B_{33}=\gamma I+\left(\mu_{3}+\delta\right) R .
\end{aligned}
$$


Table 1. Possible changes in the population

\begin{tabular}{cc}
\hline Change & Probabilty \\
\hline$\eta_{1}=(1,0,0)^{T}$ & $P_{1}=B \Delta t$ \\
$\eta_{2}=(-1,0,0)^{T}$ & $P_{2}=\mu_{1} S \Delta t$ \\
$\eta_{3}=(-1,1,0)^{T}$ & $P_{3}=f(S, I) \Delta t$ \\
$\eta_{4}=(0,-1,0)^{T}$ & $P_{4}=\mu_{2} I \Delta t$ \\
$\eta_{5}=(0,-1,1)^{T}$ & $P_{5}=\gamma I \Delta t$ \\
$\eta_{6}=(0,0,-1)^{T}$ & $P_{6}=\mu_{3} R \Delta t$ \\
$\eta_{7}=(1,0,-1)^{T}$ & $P_{7}=\delta R \Delta t$ \\
$\eta_{8}=(0,0,0)^{T}$ & $P_{8}=1-\sum_{i=1}^{7} P_{i}$ \\
\hline
\end{tabular}

It has been presented in(Allen, 1999) that the changes $\eta$ are normally distributed. Then,

$$
Y(t+\Delta t)=Y(t)+\eta \Leftrightarrow Y(t+\Delta t)=Y(t)+\mu \Delta t+\sqrt{B \Delta t} \gamma,
$$

where $\gamma_{i} \in N(0,1)$ for $i=1,2,3$. Furthermore, as $\Delta t \rightarrow 0, Y(t)$ converges strongly to the solution of the stochastic system

$$
\frac{d Y(t)}{d t}=\mu(Y(t))+\sqrt{B(Y(t))} \frac{d W(t)}{d t},
$$

where $Y(t)=(S, I, R)^{T}$ and $W(t)$ is the three-dimensional Wiener process in (Allen, 1999). The computational of (20) implies the calculation of $\sqrt{B(Y(t))}$ at each time step that is difficult.

In the next section, we derive an equivalent stochatic model which seem to be easier to implement.

\subsection{Equivalent Stochastic Differential Model}

In this section, we develop a stochastic model to examine the changes occured on each vector individually. We use the vectors defined in the previous section but here the Poisson processes $(P)$ are used to establish the different probabilities. Then, we have

$$
\left\{\begin{array}{l}
\Delta S=u_{1}-u_{2}-u_{3}+u_{4}, \\
\Delta I=u_{3}-u_{5}-u_{6}, \\
\Delta R=u_{6}-u_{7}-u_{4},
\end{array}\right.
$$

where

$u_{1} \sim P(B \Delta t), \quad u_{2} \sim P\left(\mu_{1} S \Delta t\right), \quad u_{3} \sim P(f(S, I) \Delta t), \quad u_{4} \sim P(\delta R \Delta t), u_{5} \sim P\left(\mu_{2} I \Delta t\right)$,

$u_{6} \sim P(\gamma I \Delta t), \quad u_{7} \sim \mu_{3} R \Delta t$.

We normalize the Poisson process to get

$$
\left\{\begin{array}{l}
\Delta S=B \Delta t+\sqrt{B \Delta t} \Lambda_{1}-\mu_{1} S \Delta t-\sqrt{\mu_{1} S \Delta t} \Lambda_{2}-f(S, I) \Delta t-\sqrt{f(S, I) \Delta t} \Lambda_{3}+\delta R \Delta t+\sqrt{\delta R \Delta t} \Lambda_{4}, \\
\Delta I=f(S, I) \Delta t+\sqrt{f(S, I) \Delta t} \Lambda_{3}-\mu_{2} I \Delta t-\sqrt{\mu_{2} I \Delta t} \Lambda_{5}-\gamma I \Delta t-\sqrt{\gamma I \Delta t} \Lambda_{6}, \\
\Delta R=\gamma I \Delta t+\sqrt{\gamma I \Delta t} \Lambda_{6}-\mu_{3} R \Delta t-\sqrt{\mu_{3} R \Delta t} \Lambda_{7}-\delta R \Delta t-\sqrt{\delta R \Delta t} \Lambda_{4}
\end{array}\right.
$$

where $\Lambda_{i} \in N(0,1)$ for $i=1,2, \ldots, 7$. Then, as $\Delta t \rightarrow 0$, the system (22) to the following itô stochastic differemtial equation in (Allen, 1999)

$$
\left\{\begin{array}{l}
d S=\left(B-\mu_{1} S-f(S, I)+\delta R\right) d t+\sqrt{B} d W_{1}-\sqrt{\mu_{1} S} d W_{2}-\sqrt{f(S, I)} d W_{3}+\sqrt{\delta R} d W_{4}, \\
d I=\left(f(S, I)-\mu_{2} I-\gamma I\right) d t+\sqrt{f(S, I)} d W_{3}-\sqrt{\mu_{2} I} d W_{5}-\sqrt{\gamma I} d W_{6} \\
d R=\left(\gamma I-\mu_{3} R-\delta R\right) d t+\sqrt{\gamma I} d W_{6}-\sqrt{\mu_{3} R} d W_{7}-\sqrt{\delta R} d W_{4}
\end{array}\right.
$$


System (23) can be rewritten as follows.

$$
\frac{d Y(t)}{d t}=\mu(Y(t))+G \frac{d W(t)}{d t},
$$

where $Y(t)$ and $\mu$ are the same as in system (20), $W$ is the seven-dimensional Wiener process and $G$ is defined by

$$
G=\left(\begin{array}{ccccccc}
G_{1} & -G_{2} & -G_{3} & G_{4} & 0 & 0 & 0 \\
0 & 0 & G_{3} & 0 & -G_{5} & -G_{6} & 0 \\
0 & 0 & 0 & -G_{4} & 0 & G_{6} & -G_{7}
\end{array}\right)
$$

where

$$
G_{1}=\sqrt{B}, G_{2}=\sqrt{\mu_{1} S}, G_{3}=\sqrt{f(S, I)}, G_{4}=\sqrt{\delta R}, \quad G_{5}=\sqrt{\mu_{2} I}, \quad G_{6}=\sqrt{\gamma I}, \quad G_{7}=\sqrt{\mu_{3} R}
$$

\section{Numerical Simulations}

In this section, computational simulations are given for the stochastic system (24). We use the Euler-Maruyama method to solve the SDE model (24). Let $h$ be a specified time step. The numerical method for system (24) is given by:

$$
\begin{aligned}
S^{k+1} & =S^{k}+h\left(B-\mu_{1} S-f(S, I)+\delta R\right)+\sqrt{h}\left(\sqrt{B} \eta_{1, k}-\sqrt{\mu_{1} S} \eta_{2, k}-\sqrt{f(S, I)} \eta_{3, k}+\sqrt{\delta R} \eta_{4, k}\right), \\
I^{k+1} & =I^{k}+h\left(f(S, I)-\mu_{2} I-\gamma I\right)+\sqrt{h}\left(\sqrt{f(S, I)} \eta_{3, k}-\sqrt{\mu_{2} I} \eta_{5, k}-\sqrt{\gamma I} \eta_{6, k}\right), \\
R^{k+1} & =R^{k}+h\left(\gamma I-\mu_{3} R-\delta R\right)+\sqrt{h}\left(\sqrt{\gamma I} \eta_{6, k}-\sqrt{\mu_{3} R} \eta_{7, k}-\sqrt{\delta R} \eta_{4, k}\right),
\end{aligned}
$$

for $k=0,1,2, \ldots$ until the maximum time is reached.

Here, $\eta_{i, k}$ for $i=1,2, \ldots, 9$ and $k=0,1,2, \ldots$ are normally distributed numbers with zero mean and unit variance.

Here, one case of computational simulation were studied. In this case $R_{0}<1$. In the computation, the functions $f$ is chosen as follows $f(S, I)=\beta S I$ (mass action).

The parameters values used are given as: $B=14$ (recruitment) $\mu_{1}=0.014, \delta=0.002, \mu_{2}=0.014, \gamma=0.05, \mu_{3}=0.2$. The initial values of the population sizes are taken as $S(0)=600, I(0)=1300, R(0)=400$. The time step $h$ is chosen as $h=0.3$ year and the final time was taken as 300 days. These figures are produced by Matlab.
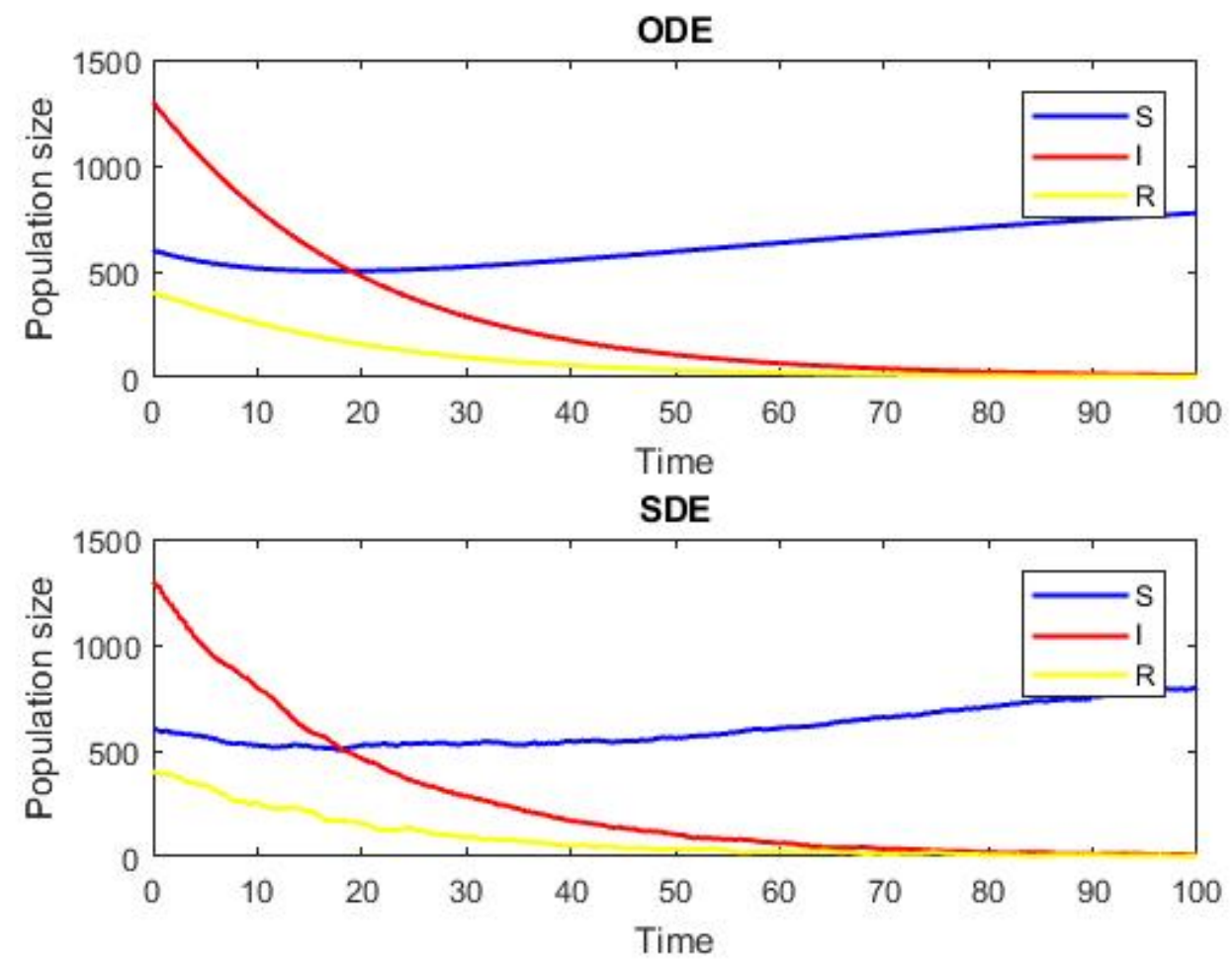

Figure 1. Deterministic and equivalent stochastic models for $R_{0}<1$ 

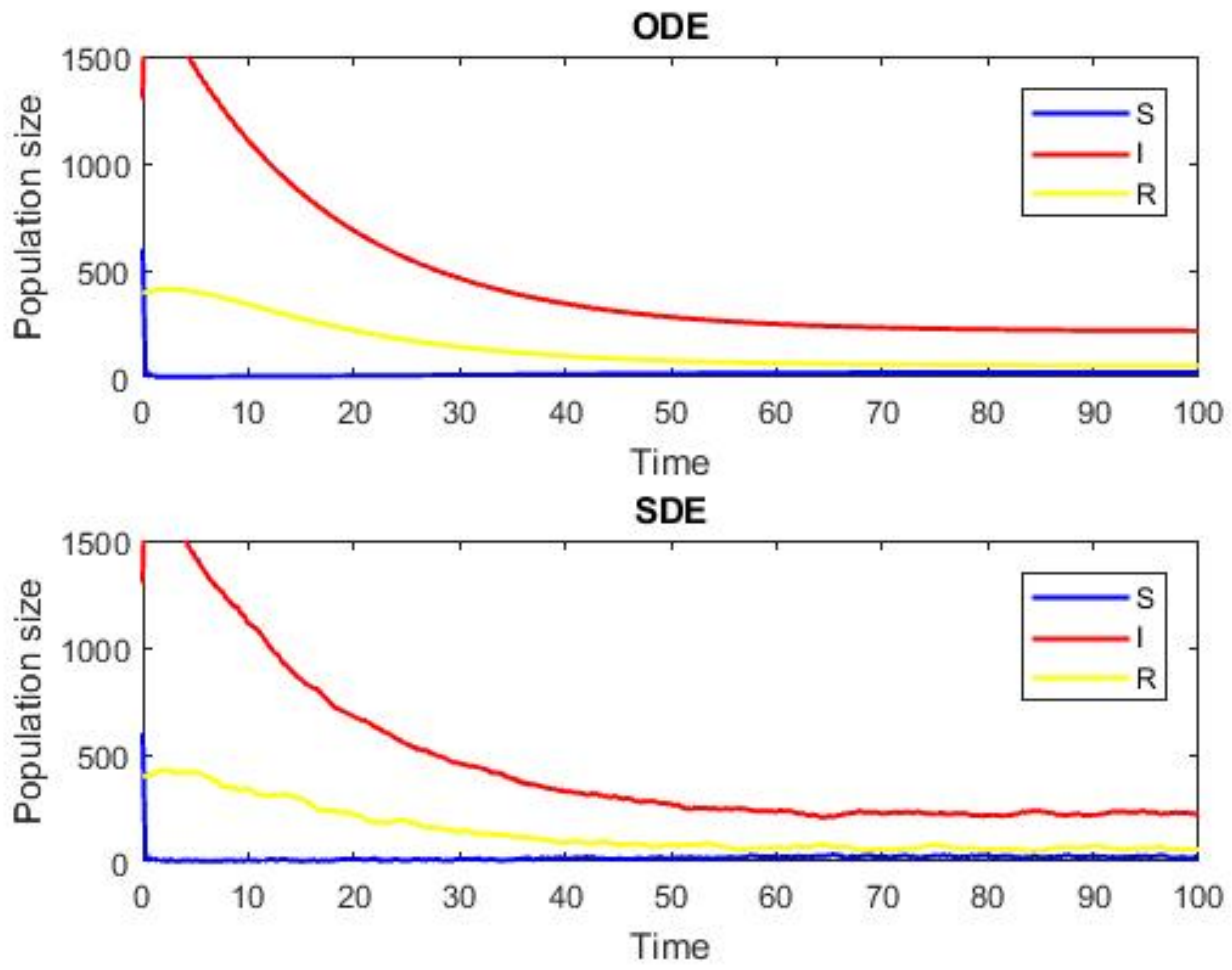

Figure 2. Deterministic and equivalent stochastic models for $R_{0}>1$

Figure 1 illustrates the deterministic model (1) and the equivalent stochastic model (24) when $R_{0}<1$. We can see that, in the Figure 1 the trajectory of deterministic and stochastic graphs are approximately the same behaviour. Indeed, the infected extinction is effective if $R_{0}<1$.

Figure 2 illustrates the deterministic model (1) and the equivalent stochastic model (24) when $R_{0}>1$. In Figure 2, we can see that the determinsitic graph are similar to those of the stochastic graph, the susceptible decrease is effective for this two models when $R_{0}<1$.

\section{Conclusion}

In this paper, an SIR epidemic model with the general incidence function is derived. In the first hand, the global behaviour of the model system was studied. We proved that, if $R_{0} \leq 1$ holds, then the disease-free equilibrium is globally asymptotically stable, Which implies that the disease fades out from the population. If $R_{0}>1$, then there exists a unique endemic equilibrium which is globally asymptotically stable, and this implies that the disease will persist in the population.

In a second part of this work, we construct a stochastic models derive from the deterministic models. The behavior of the stochastic models are studied. Computational simulations were presented to make comparison between deterministic and equivalent stochastic models. The behavior of the detrministic and equivalent stochastic models are approximately the same.

\section{References}

Allen, E. J. (1999). Stochastic Differential equations persistence time of two interacting populations, Dynamics of continuous, Discrete and Impulsive Systems, 5, 271-281.

Allen, L. J. S. (2003). An introduction to Stochastic Processes with Applications to Biology. Printice-Hall, Englewood Cliffs.

Allen, E. J., \& Victory, H. D. (2003). Modelling and Simulation of a Schistosomiasis infection with Biological Control. Acta Tropica, 87(2), 251-267. https://doi.org/10.1016/s0001-706x(03)00065-2

Connell, C. M.(2010). Global stability for an SIR epidemic model with delay and nonlinear incidence, Nonlinear Anal.RWA, 11, 3106-3109. https://doi.org/10.1016/j.nonrwa.2009.11.005 
Gao, S., Chen, L., Nieto, J. J., \& Torres, A. (2006). Analysis of a delayed epidemic model with pulse vaccination and saturation incidence, Vaccine, 24, 35-36, 6037-6045. https://doi.org/10.1016/j.vaccine.2006.05.018

Gard, T. C. (1988).Introduction to Stochastic Differential Equations, Marcel Dekker, New York. https://doi.org/10.1002/zamm.19890690808

Graunt, J. (1662). Natural and political observations made upon the bills of mortality. Roycroft and Dicas: London.

Guiro, A., Ngom, D., \& Ouedraogo, D. (2017). Global stability for a class of discrete Schistosomiasis models with general incidence Advances in Difference Equations. 1-16. https://doi.org/10.1186/s13662-017-1174-6

Guiro, A., Ouedraogo, D., \& Ouedraogo, H. (2018). Stability analysis for a discrete SIR epidemic model with delay and general nonlinear incidence function. Applied Mathematics, 9, 1039-1054. https://doi.org/10.4236/am.2018.99070

Jiang, Z., \& Wei, J. (2008). Stability and bifurcation analysis in a delayed SIR model, Chaos, Solitons and Fractals, 35, 609-619, https://doi.org/10.1016/j.chaos.2006.05.045

Kermack, W. O., \& Mckendric, A. G. (1927). Contribution to the mathematical theory of epidemics, Proc. R. Soc. Lond. Ser. A, 115, 700-721.

Kyrychko, Y., \& Blyuss, B. (2005). Global properties of a delayed SIR model with temporary immunity and nonlinear incidence rate. Nonlinear Anal.: Real World Appl., 6, 495-507. https://doi.org/10.1016/j.camwa.2010.03.009

Lakshmikantham, V., Leela, S., \& Martynyuk, A. A. (1989). Stability Analysis of Nonlinear Systems. Marcel Dekker, New York. https://doi.org/10.1142/1192

Lasalle, J. P. (1976). The Stability of Dynamical Systems. SIAM, Philadelphia. https://doi.org/10.21236/ADA031020

Li, G., Wang, W., Wang, K., \& Jin, Z. (2007). Dynamic behavior of a parasite-host model with general incidence. J. Math. Anal. Appl., 631-643. https://doi.org/10.1016/j.jmaa.2006.09.015

Wei, C., \& Chen, L. (2008). A delayed epidemic model with pulse vaccination. Discrete Dynamics in Nature and Society, Vol. 2008, Article ID 746951. https://doi.org/10.1155/2008/746951

Zhang, F., Li, Z. Z., \& Zhang, F. (2008). Global stability of an SIR epidemic with constant infectious period, Applied Mathematics and Computation, 199, 285-291. https://doi.org/10.1016/j.amc.2007.09.05

Zhang, J. Z., Jin, Z., Liu, Q. X., \& Zhang, Z. Y. (2008). Analysis of a delayed SIR model with nonlinear. Discrete Dynamics in Nature and Society, Vol. 2008, Article ID 66153. https://doi.org/10.1155/2008/636153

Zhou, Y., \& Liu, H. ( 2003). Stability of periodic solutions for an SIS model with pulse vaccination, Mathematical and Computer Modelling, 38, 299-308. https://doi.org/10.1016/S0895-7177(03)90088-4

\section{Copyrights}

Copyright for this article is retained by the author(s), with first publication rights granted to the journal.

This is an open-access article distributed under the terms and conditions of the Creative Commons Attribution license (http://creativecommons.org/licenses/by/4.0/). 